\title{
CLOSED LOOP IDENTIFICATION BASED ON QUANTIZATION
}

\section{Wang*, N.F. Thornhill ${ }^{+}$and B. Huang $\#$}

*Centre for Process Systems Engineering, Imperial College of Science and

Technology, Prince Consort Road, London SW7 2BY.

${ }^{+}$Centre for Process Systems Engineering, Department of Electronic and

Electronic Engineering, Torrington Place, University College London, London WCIE 7JE (corresponding author).

\#Department of Chemical and Materials Engineering, University of Alberta, Edmonton, Alberta, Canada T6G 2 G6.

\begin{abstract}
This paper proposes a new closed-loop identification scheme for a singleinput-single-output control loop. It is based upon a quantizer inserted into the feedback path. The quantizer can be used to generate an equivalent persistently exciting signal with which the well known two-stage and/or two-step method can be used directly. Simulation examples and an experimental demonstration are used to illustrate the proposed scheme. Copyright (C) 2002 IFAC
\end{abstract}

Keywords: Chemical industry; closed-loop identification; process control; quantization; time-series analysis.

\section{INTRODUCTION}

\subsection{Closed-loop identification}

A process model is necessary for model-based process control. The purpose of closed loop identification (CLID) is to identify a process model while the process is still under feedback control (MacGregor and Fogal, 1995). CLID might be necessary because the system is unstable in open loop or the system contains inherent feedback mechanisms (Ljung, 1987). "CLID is also motivated by the fact that many industrial processes have already in place one or more loops that cannot (or better not) be removed for safety and/or economic reasons" (Jorgensen and Lee, 2001).

External excitation is a dither signal injected into the closed-loop system to excite the process for the purpose of system identification. A single-input single-output (SISO) closed-loop system (adopted from MacGregor and Fogal, 1995) illustrates the material to be presented in this paper. It provides a general structure for CLID with external excitation. The excitation is a dither signal introduced at $d$ as shown in figure 1.
In figure $1, G_{0}(q)$ represents the true process, and the disturbance $v(t)=H_{0}(q) a(t)$ represents the effect of all unmeasured process disturbances on the measured output $y(t), q$ is the forward shift operator. $C(q)$ is the feedback controller, and the set-point $y_{s p}(t)$ and the 'dither' signal $d(t)$ are input signals that may be injected to aid the identification. In this paper $y_{s p}(t)=0$ is considered, that is to say, $d(t)$ is the only input signal. The task is to use $y(t)$ and $u(t)$ or $d(t)$ to identify the process.

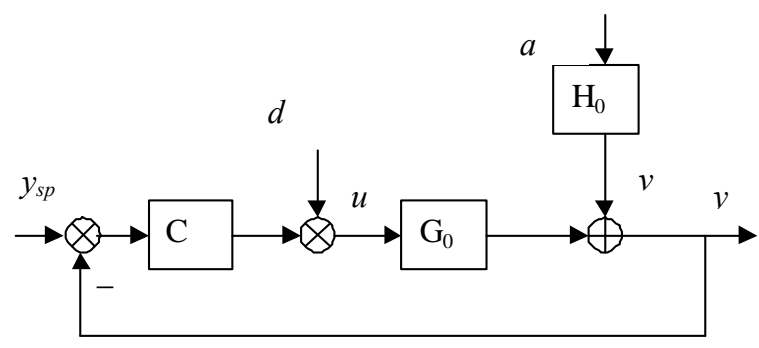

Fig. 1 Closed-loop system 
Among the different closed-loop identification methods, the two-stage and two-step methods (Van den Hof and Schrama, 1993; Huang and Shah, 1997) have attracted much attention. Esmaili et. al., (2000) discussed the asymptotic and finite data behaviour of some closed-loop identification methods including the two-step method by Huang and Shah.

\subsection{Closed-loop identification with a quantizer}

A quantizer operates on the amplitude axis. Its output is constrained to be one of several discrete values, whichever is closest to the true measurement.

A quantizer inserted into the feedback path may be used to generate an excitation signal that is equivalent to the persistently exciting external excitation required by system identification. The twostage method by Van den Hof and Schrama (1993) and/or the two-step method by Huang and Shah (1997) can be used directly for identification.

The major benefit of the proposed CLID scheme is that there is no requirement for special design of an external excitation. Rather, the quantization interval is adjusted. The quantizer can be implemented by hardware or by software. When the identification is complete, the quantization interval is made negligibly small.

The use of a special quantizer (mid-rise quantizer and mid-step quantizer) was reported in Goodwin and Welsh (1999) and Welsh and Goodwin (1999). The quantizer was inserted after the controller and before the process for the purposes of multiple-inputmultiple-output (MIMO) autotuning. The loops were required to oscillate at the critical frequency of the process as in relay tuning and test signals were injected to achieve identification at other frequencies. The authors demonstrated that the special quantizers had benefits over traditional relay tuning, for example that the process would stay under control in the presence of a large disturbance during the identification procedure.

The focus of this paper is to use the quantizer itself as the source of external excitation. There is no need to cause the control loop to oscillate and consequently the upset to the loop is minimized.

\subsection{Layout}

The paper is laid out as follows. Section 2 presents theory relevant to closed loop identification with a quantizer. Section 3 presents the two-stage method and the two-step method. Other methods including simulation and experimental methods are also discussed. Simulation and experimental results are presented in section 4 together with the discussions. Conclusions are given in section 5.

\section{THEORY}

\subsection{Closed-loop Identifiability}

A fundamental problem with closed-loop data is the correlation between the unmeasurable noise and the process input. When feedback is used, the input and the noise will be correlated because the input $u$ is determined from the process variable, which contains the noise. That is the reason why some open loop identification methods fail to work with closed-loop data (Forssell and Ljung, 1999). It is necessary to break the dependency between the process input and the process noise.

MacGregor and Fogal (1995) discussed closed-loop identifiability. They showed that if data from purely feedback operations are used, then nonparametric methods in which a model is described by a curve, function or a table yield no information on the true system, but only the inverse of the controller dynamics. Under the same conditions, the parametric methods (in which the model is expressed as a transfer function in continuous-time form or discretetime form) can identify the true system only when the order of the controller is greater than or at least equal to the order of the process. In Gustavsson et. al. (1977), this was called System Identifiability (SI).

Bartree and McFarlane (1998) studied the identification of process models using routine operating data with simulation examples in a fluid catalytic cracking unit. They showed that with routine data (from systems satisfying SI), only when the true system structure is known, can an adequate model be identified with parametric methods. The conclusion is the same as that in MacGregor and Fogal (1995). This indicates that routine or archived data have limited practical use in closed-loop system identification.

Closed-loop identification can be accomplished in two ways (MacGregor and Fogal, 1995; Bartee and McFarlane, 1998):

- By injecting an independent, persistently exciting signal into the feedback loop;

- By switching between two or more feedback controllers.

Either of these will guarantee that necessary and sufficient conditions for identifiability be satisfied. This condition is called Strong System Identifiabilty (SSI) (Gustavsson et. al., 1977).

Therefore injecting external excitation into a system causes it to be SSI. The injection of an independent signal into the loop provides the user with a greater flexibility for designing the identification experiment to achieve the desired objectives.

It will be shown in this paper that when the quantization interval is within the range suggested, the quantizer error excitation is equivalent to a persistently exciting external signal. 


\subsection{Quantizer definition}

Goodwin and Welsh (1999) and Welsh and Goodwin (1999) defined two special quantizers (mid-rise quantizer and mid-step quantizer) for the purpose of MIMO autotuning. The two quantizers referred to above can be generated as special cases of a quantizer with three parameters: quantization interval (qi), quantization level $(q l)$ and hysteresis width $(\varepsilon)$. The three-parameter implementation is practical since the setpoint of a control loop is in reality somewhere between $4 \mathrm{~mA}$ and $20 \mathrm{~mA}$. By adjusting the parameters $q l$ and/or $q i$, a quantizer can be made midrise or mid-step as desired for a process at, say, a setpoint of $10.5 \mathrm{~mA}$. Throughout this paper, the new quantizer functionally acted like a mid-step quantizer. The hysteresis width $(\varepsilon)$ was set to zero since it did not make difference in generation of the excitation signal.

\subsection{Key issues when aquantizer is used}

For a given process, there are conflicts in choosing quantization interval when a quantizer is inserted. If the quantization interval is small, the quantization excitation can be shown to be white (therefore guaranteed persistently exciting) and uncorrelated to the process disturbance, but then the signal-to-noise ratio is not large enough to do CLID. If the quantization interval is larger, the quantization excitation will be large enough for CLID, but correlation then exists between the quantization excitation and the process disturbance. This will also endanger the CLID. The challenge of determination of the appropriate quantization interval is addressed in section 4.1.

\section{METHODS}

3.1 Adaptation of the two-stage and two-step methods Consider a system with the excitation in the feedback path (figure 2):

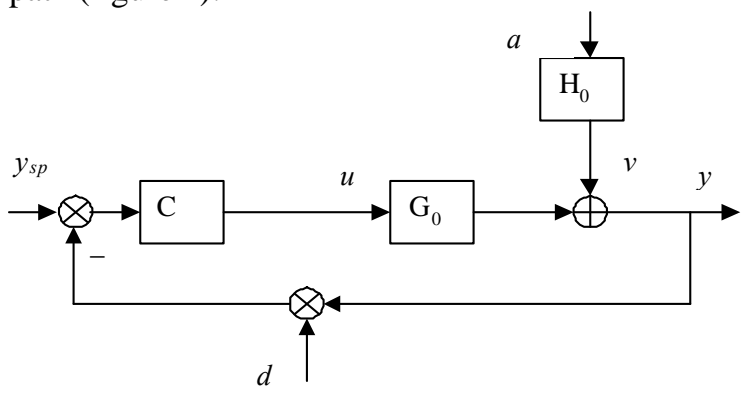

Fig. 2. Closed-loop system with excitation in the feedback path.

$$
\begin{aligned}
& u(t)=\frac{-C(q)}{1+C(q) G_{0}(q)} d(t)-\frac{C(q)}{1+C(q) G_{0}(q)} v(t) \\
& y(t)=\frac{-C(q) G_{0}(q)}{1+C(q) G_{0}(q)} d(t)+\frac{1}{1+C(q) G_{0}(q)} v(t)
\end{aligned}
$$

From (1) and (2)

$$
\begin{aligned}
& u(t)=-C(q) S_{0}(q) d(t)-C(q) S_{0}(q) H_{0}(q) a(t) \\
& y(t)=-C(q) G_{0}(q) S_{0}(q) d(t)+S_{0}(q) H_{0}(q) a(t)
\end{aligned}
$$

In the two-stage method by Van den Hof and Schrama (1993), the sensitivity function $S_{0}$ can be obtained from excitation $d$ and process input $u$. Then a noise-free process input $u^{d}$ can be simulated from excitation $d$ and the sensitivity function, the process $G_{0}$ is calculated from the noise-free process input $u^{d}$ and the process output.

$$
\begin{aligned}
& u(t)=-C(q) S_{0}(q) d(t)-C(q) S_{0}(q) H_{0}(q) a(t) \\
& u^{d}(t)=-C(q) S_{0}(q) d(t) \\
& y(t)=G_{0}(q) u^{d}(t)+S_{0}(q) H_{0}(q) a(t)
\end{aligned}
$$

The two-step method by Huang and Shah (1997) also gives the sensitivity function from excitation $d$ and process input $u$, from which $G_{0}$ may then be determined.

$$
\begin{aligned}
& u(t)=-C(q) S_{0}(q) d(t)-C(q) S_{0}(q) H_{0}(q) a(t) \\
& \frac{y(t)}{-C(q) S_{0}(q)}=G_{0}(q) d(t)+\frac{H_{0}(q) a(t)}{-C(q)}
\end{aligned}
$$

When the excitation position is in the feedback path, the two-step and two-stage methods have the same procedure, the only difference is that $-C(q) S_{0}(q)$ instead of $S_{0}(q)$ is identified from $u(t)$ and $d(t)$ in the first step and that $-C(q) S_{0}(q)$ instead of $S_{0}(q)$ is then used in the second step.

\subsection{The proposed CLID scheme}

A quantizer is inserted in the feedback path to generate the equivalent excitation whose characteristics have been discussed in the theory section. Therefore the adapted method as shown in equations [5-7] or [8-9] can be used directly.

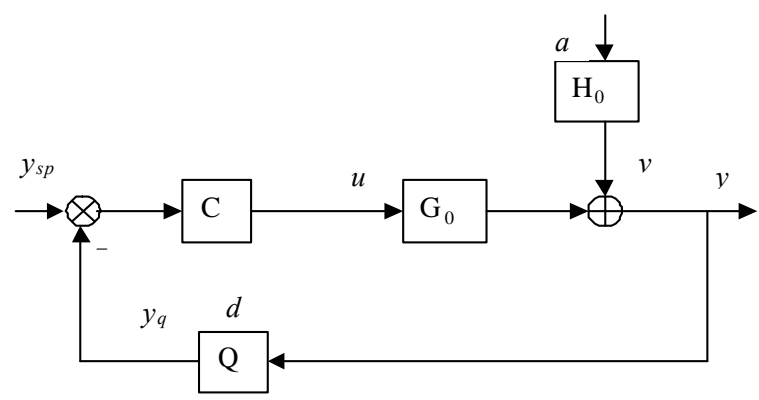

Fig. 3. The proposed CLID scheme

The procedures for the proposed CLID scheme are as follows:

- The process is run as normal (with quantization interval very small, for example 0.005 , equivalent to 12 bit $\mathrm{A} / \mathrm{D})$. The standard deviation of process output $\sigma_{y}$ is calculated; 
- A specific quantization interval (for example $1.5 \times \sigma_{y}$ in the range of $\left(\sigma_{y} \sim 2 \sigma_{y}\right)$ is chosen.

The process is run with this quantization interval. The quantizer error excitation $d=y_{q}-y$ can be guaranteed to be good enough to do identification, as shown in section 4;

- The two-stage and/or two-step method can be used directly.

\subsection{Simulation method}

To demonstrate the theoretical analysis and the methods discussed above, a second order ARMAX model presented in Huang and Shah, (1997) was chosen. The transfer function is:

$$
\begin{aligned}
& \left(1-0.7859 q^{-1}+0.3679 q^{-2}\right) y(t)=(0.3403+ \\
& \left.0.2417 q^{-1}\right) u(t-1)+\left(1-0.8 q^{-1}+0.12 q^{-2}\right) a(t)
\end{aligned}
$$

A unit feedback control law is implemented in this simulation (figure 4). The white noise $a(t)$ is applied. The number of sampled data points in the simulation was 10000 .

Correlation and signal-to-noise ratio tests: The simulation shown in figure 4 was used for the purpose of exploring the characteristics of the quantizer excitation under different quantization intervals. In this simulation, a white noise with variance $\sigma_{a}^{2}=1$ was applied and fixed throughout different tests. The standard deviation of the process output $\sigma_{y}$ was measured when the quantization interval was 0.005 . There were all together 30 tests with $q i=0.1,0.2, \ldots 3.0$ (see figure 7 ).

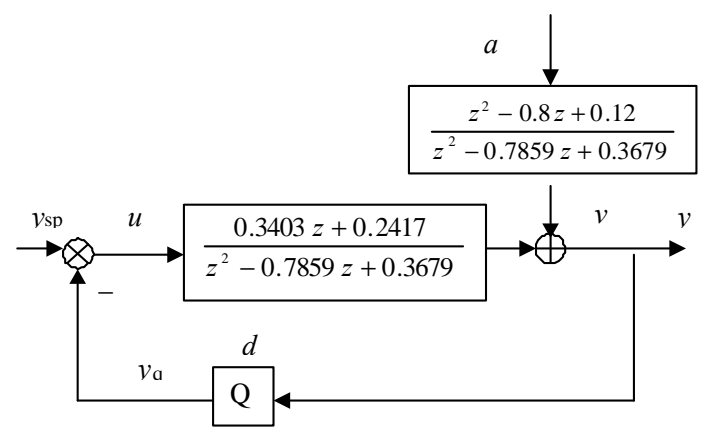

Fig. 4. Simulation for CLID with a quantizer

The signal to noise ratio was the ratio between the variances of the quantizer excitation $y_{q}-y$ and the noise:

$$
S N R=\frac{\operatorname{Var}\left(y_{q}-y\right)}{\operatorname{Var}(v)}
$$

CLID with a quantizer (simulation): To explore the proposed method under different disturbances and different quantization intervals, the following three cases (Table 1) were designed and tested in simulation. $\underline{\text { Table 1. Simulation conditions }}$

\begin{tabular}{lrrr} 
& Case 1 & Case 2 & Case 3 \\
\hline Variance of disturbance & 0.010 & 0.010 & 1.000 \\
$\begin{array}{l}\text { Disturbance equivalent } \\
\text { to 4-20mA }\end{array}$ & 0.100 & 0.100 & 1.000 \\
$\begin{array}{l}\text { Standard deviation of } \\
\text { process output when } \\
q i=0.005\end{array}$ & 0.137 & 0.137 & 1.373 \\
$\begin{array}{l}\text { Quantization interval } \\
\text { (qi) for identification }\end{array}$ & 0.137 & 0.2055 & 1.373 \\
$\begin{array}{l}q i \text { for } \\
\text { identification/ } \sigma_{y}\end{array}$ & 1 & 1.5 & 1 \\
$\begin{array}{l}\text { Quantization interval } \\
\text { equivalent to A/D bit }\end{array}$ & 7 & 6 & 3 or 4 \\
\hline
\end{tabular}

\subsection{Experimental method}

An experimental apparatus (as shown in figure 5) was used to evaluate the proposed scheme. It was a doubled-walled stirred glass tank. This system is located in the University of Alberta computer process control laboratory. The aim was to determine the open loop temperature dynamics from closed loop data.

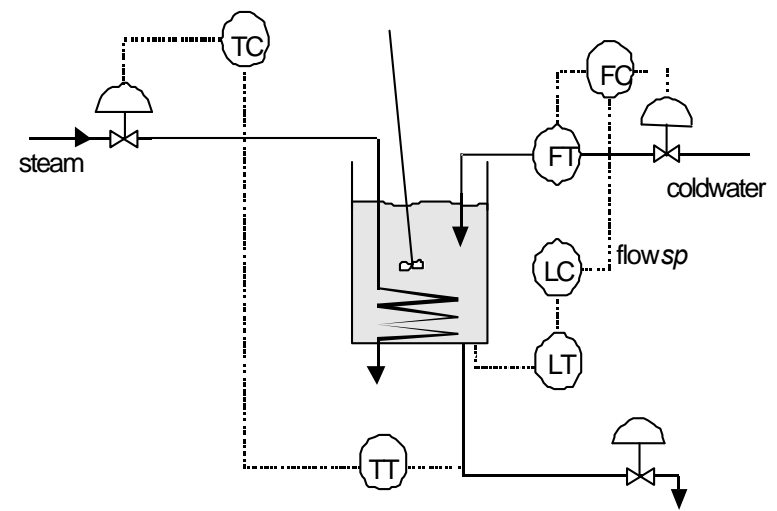

Fig. 5. The experimental apparatus

The level of the tank was always controlled to be $12 \mathrm{~mA}$ on a $4-20 \mathrm{~mA}$ scale (that is in the middle of the tank) during the experiment. The manipulated variable for the temperature control loop was the steam flow and controlled variable was the temperature of the water leaving the tank. A PI controller in discrete form was applied. The setpoint for the temperature loop was $10.5 \mathrm{~mA}\left(41.5{ }^{\circ} \mathrm{C}\right)$. Compressed air blown from the bottom of the tank caused bubbles to provide a natural disturbance.

An open loop test was conducted with a random binary signal as excitation. This open loop identification result may be viewed as the true process. The test for CLID with a quantizer was to determine that same result. The proposed CLID scheme was conducted under the conditions given in Table 2. 


\section{Table 2. experimental conditions}

Standard deviation of process output $\quad 0.0657$ when $q i=0.005$

Quantization interval ( $q i)$ for

identification

$q i$ for identification $/ \sigma_{y}$

Quantization interval equivalent to

\section{A/D bit}

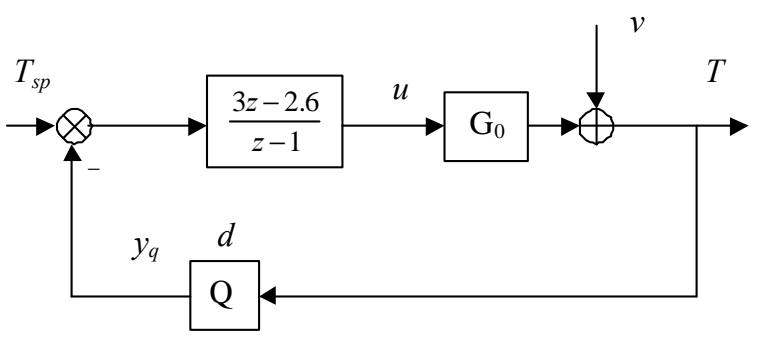

Fig. 6. Block diagram for closed-loop identification of temperature loop

\section{RESULTS AND DISCUSSIONS}

\subsection{Simulation Results and Discussions}

Correlation and signal-to-noise ratio: The result for the simulation (subsection 3.3) is shown in figure 7, in which the signal refers to quantization error.
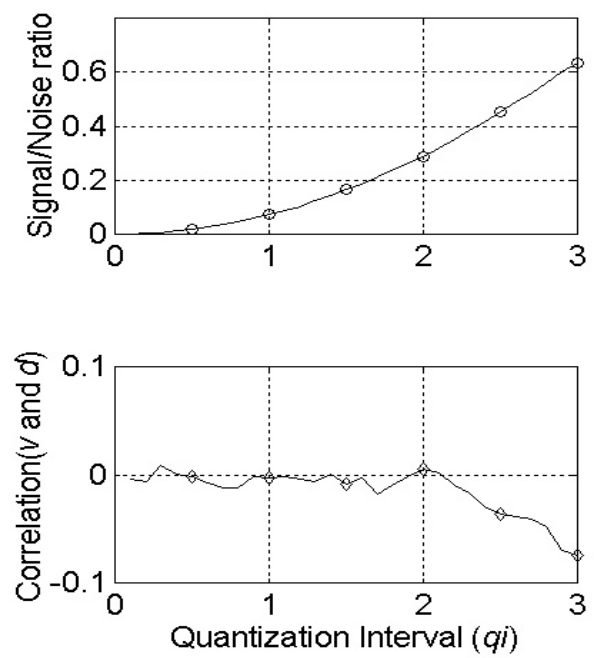

Fig. 7: Correlation between the quantizer excitation and disturbance (above) and the signal-to-noise ratio for the given ARMAX process (below).

The standard deviation of the process output $\sigma_{y}$ was 1.373 when the quantization interval was 0.005 . From figure 7, the range of quantization interval from 1.4 to 2.8 is of special interest because the signal-tonoise ratio is large enough for some CLID methods (for example two stage and two-step methods) and the correlation between the excitation and noise/disturbance is negligible. This range (from 1.4 to 2.8) corresponds to $\sigma_{y}$ to $2 \sigma_{y}$ approximately. The simulation suggests that when the quantization interval is within this range, the identified model accuracy can be guaranteed.

Simulation of CLID with a quantizer: Nyquist plots identified in simulations using CLID with a quantizer (simulation conditions indicated in table 1) are shown in figure 8 . The panels (top to bottom) in figure 8 corresponds to cases 1 to 3 in table 1 .
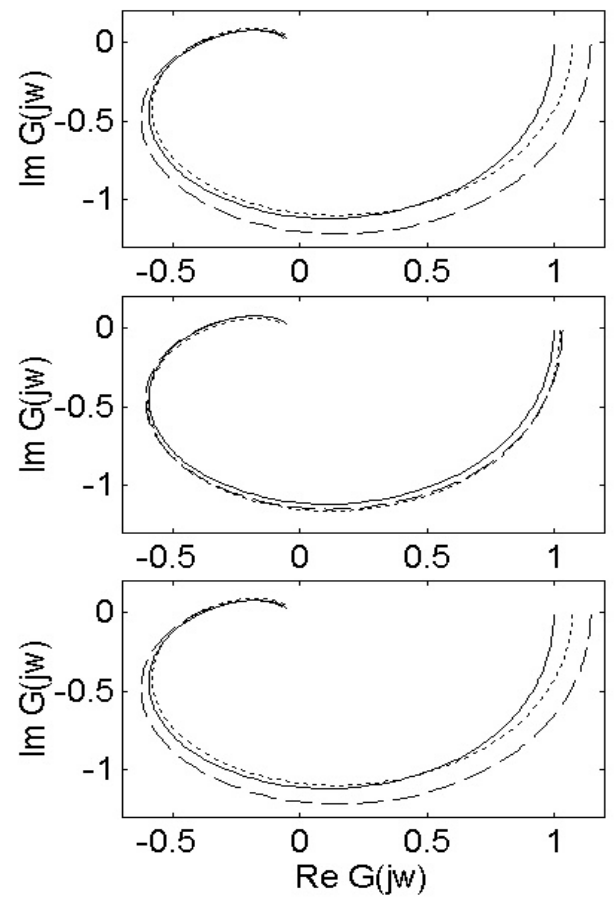

Fig. 8. Results for simulations in table 1 (Solid: true process; Dotted: Huang and Shah,1997; Dashed: Van den Hof and Schrama, 1993)

From table 1, Case 1 and Case 2 have the same variance of disturbance (0.010). The only difference is the quantization interval applied ( $\sigma_{y}$ in Case 1 and $1.5 \times \sigma_{y}$ in Case 2). The second panel of figure 8 , compared with the first panel of figure 8, shows improved accuracy of the identified model. This is because the signal-to-noise ratio increased in Case 2 due to the increased quantization interval.

Case 1 and Case 3 have different variance of disturbance ( 0.010 vs 1.000$)$. However, in both cases, the ratio of the quantization interval and the standard deviation $\sigma_{y}$ is the same, that is $q i=\sigma_{y}$. From the top panel and the bottom panel of figure 8 , they have achieved almost the same identification model accuracy. This indicates the proposed scheme is suitable for both small disturbance and large disturbance. 


\subsection{Experimental Results and Discussions}

The result from experimental demonstration of CLID with a quantizer (subsection 3.4) is shown in figure 9.
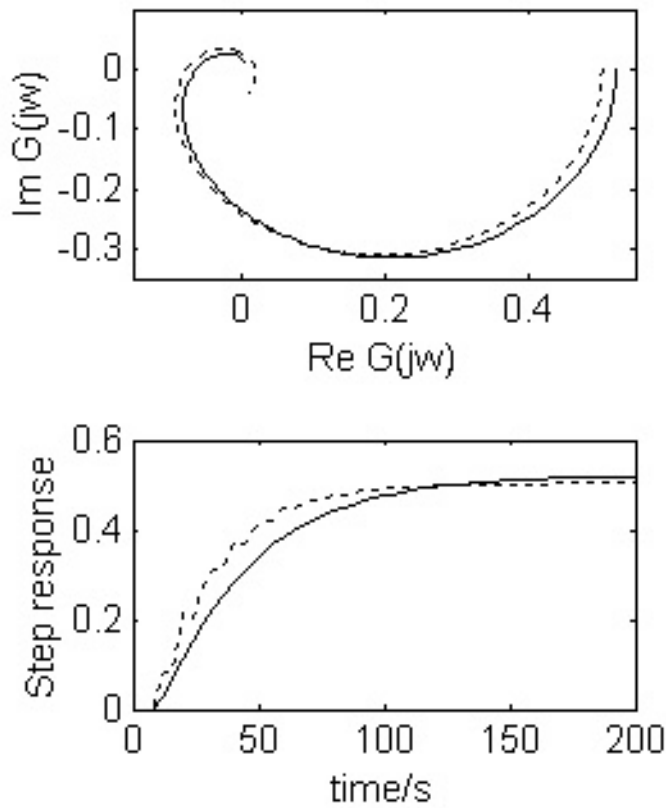

Fig. 9. Experimental result (dotted) versus open loop identification (solid) for temperature loop.

From the top panel of figure 9, the closed-loop identification result is well matched with the open loop result in both low frequency and the medium frequency, with some mismatch in high frequency. However, in general, the most important part of the model is the frequency band from zero to crossover frequency. The comparison of the open loop result and the closed-loop identification result is also plotted in the bottom panel of figure 9 in the form of time-domain step response. The time delay is perfectly caught in the closed-loop identification. The steady-state gains are very close to each other. The high frequency mismatch is also indicated in the transient process that closed-loop identification result has a faster dynamics.

\section{CONCLUSION}

In this paper, a new scheme for CLID has been proposed. The benefit of the proposed CLID with a quantizer inserted in the feedback path is that it provides a convenient way to generate a persistently exciting signal with quantizer. Using the two-stage and/or two-step method, it was able to successfully identify process models without additional external excitations.

With this scheme, the disadvantage of switching off the controller in relay-based identification can be avoided and there is no need to cause the control loop to oscillate. Simulation examples with a discrete ARMAX model and an experimental demonstration indicate the applicability of this strategy.

\section{ACKNOWLEDGEMENTS}

Meihong Wang gratefully acknowledges the financial support of the Centre for Process Systems Engineering, Imperial College of Science, Technology and Medicine, London. The UK authors thank the CPC group of the Department of Chemical and Materials Engineering at the University of Alberta for use of pilot plant and facilities.

\section{REFERENCES}

Bartree, J.F., and R.C. McFarlane (1998). Identification of linear systems operating in closed loop, Proceedings of IChemE: Advances in Process Control, Swansea, 2-3 Sept. 1998.

Esmaili, A., J.F. MacGregor, and P.A. Taylor (2000). Direct and two-step methods for closed-loop identification: a comparison of asymptotic and finite data set performance, J. Proc. Cont., 10, 525-537.

Forssell, U., and L. Ljung (1999). Closed-loop identification revisited, Automatica, 35, 12151241.

Goodwin, G.C., and J.S. Welsh (1999). Analysis of a novel method of autotuning a multivariable plant based on quantization, Proceedings of American Control Conference, San Diego, California.

Gustavsson, I., L. Ljung, and T. Soderstrom (1977). Identification of processes in closed loop Identifiability and accuracy aspects, Automatica, $13,59-75$.

Huang, B., and S.L. Shah (1997). Closed-Loop identification: a two step approach, J. Proc. Cont., 7, 425-438.

Ljung, L. (1987). System Identification: theory for the user, Englewood Cliffs, NJ: Prentice-Hall.

MacGregor, J.F., and D.T. Fogal (1995). Closed-loop identification: the role of the noise model and prefilters, J. Proc. Cont., 5, 163-171.

Jorgensen, S.B., and J.H. Lee (2001). Recent advances and challenges in process identification, Proceedings of Sixth International Conference on Chemical Process Control, AIChE Symposium Series, Tucson, Arizona, USA.

Van den Hof, P.M.J., and R. Schrama (1993). An indirect method for transfer function estimation from closed loop data, Automatica, 29, 15231527.

Welsh, J.S. and G.C. Goodwin (1999). A novel mechanism for autotuning based on quantization, Proceedings IFAC 14th World Congress, Beijing China. 